\title{
Stability of cnoidal wave frequency combs in microresonators
}

\author{
Zhen $\mathrm{Qi}^{1}$, Shaokang Wang ${ }^{1}$, Jose A. Jaramillo-Villegas ${ }^{2}$, Minghao $\mathbf{Q i}^{2}$, Andrew M. \\ Weiner $^{2}$, Giuseppe D'Aguanno ${ }^{3}$, Curtis R. Menyuk ${ }^{1}$ \\ 1. University of Maryland at Baltimore County, 1000 Hilltop Circle, Baltimore, MD 21250, USA \\ 2. Purdue University, West Lafayette, IN 47907, USA \\ 3. University of Texas at Austin, 110 Inner Campus Drive, Austin, TX 78712, USA \\ zhenqi1@umbc.edu
}

\begin{abstract}
We determine the regions in the parameter space of microresonators where cnoidal waves (Turing rolls) are stable. Solitons are included as a special limit. We identify conditions to efficiently obtain broadband frequency combs.
\end{abstract}

OCIS codes: (230.5750) Resonators; (190.5530) Pulse propagation and temporal solitons; (190.4380) Nonlinear optics, four-wave mixing.

Broadband frequency combs have a wide range of potential applications-ranging from basic science and metrology to military and medical engineering [1]. Due their small size, weight and power, and the large frequency spacing of their comb lines, microresonator-based optical frequency combs have attracted a large amount of attention in the past decade [2]. Within the past year, $1 f$-2 $f$ locking of these combs has been demonstrated [3].

Work to date on broadband combs has focused almost entirely on solitons, but it has long been known that solitons are a special limit of a much larger family of periodic waveforms that are referred to alternatively as cnoidal waves [4] or Turing rolls [5]. There are good reasons for focusing attention on this larger family. Solitons are difficult to access and use the pump inefficiently. By contrast, work to date indicates that most members of the cnoidal wave family are easier to access and use the pump far more efficiently than do solitons $[4,5]$. While in most cases they are more narrowband than solitons, we will show here that they can be broadband, while also being robustly accessible.

It has been shown that propagation in microresonators can be modeled using the Lugiato-Lefever equation (LLE) [6], which in normalized form may be written,

$$
\frac{\partial \psi}{\partial t}-i \frac{\partial^{2} \psi}{\partial x^{2}}-i|\psi|^{2} \psi+(1+i \Delta) \psi-F=0
$$

where $\psi$ is the normalized complex amplitude of the intracavity field, $t$ is the time, $x$ is the normalized retarded distance, $\Delta$ is the normalized detuning, and $F$ is the normalized pump amplitude. A third parameter $L$, which is the normalized repetition period of the intracavity field, is needed to completely characterize the parameter space. In experimental systems that have studied broadband combs in microresonators, $-10 \lesssim \Delta \lesssim 10,0 \lesssim|F|^{2} \lesssim 10$, and $30<L<200$.

In order to determine the regions in the $\Delta-|F|^{2}-L$ parameter space where stable cnoidal wave solutions exist, we use boundary tracking algorithms that we previously developed to study lasers [7]. In this approach, we begin with a stable stationary solution of Eq. (1), which we can find—for example—by solving the evolution equations. We then follow the stationary solution $\psi_{s}[x ; \Delta, F, L]$ as the parameters vary by setting $\partial \psi / \partial t=0$ and solving the nonlinear root-finding
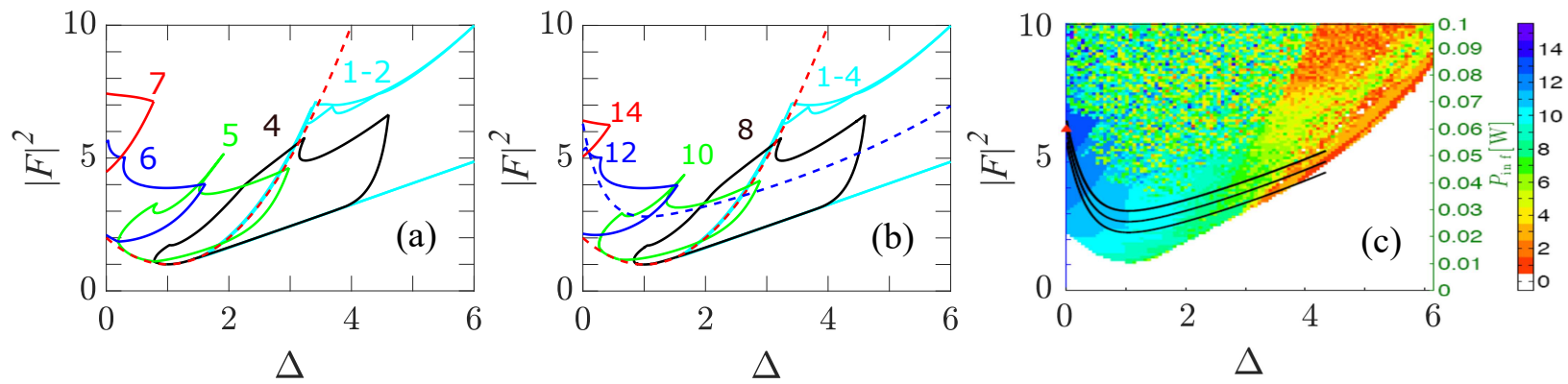

Fig. 1. Stability chart of the cnoidal waves at (a) $L=25$ and (b) $L=50$. For clarity, only some of the periodicities are shown. The numbers labeling the contours in (a) and (b) denote the number of periods $N_{\text {per }}$. The stability regions for $N_{\text {per }}=1-2$ when $L=25$ and for $N_{\text {per }}=1-4$ for $L=50$ almost completely overlap. The case $N_{\text {per }}=1$ corresponds to solitons. Below the red-dashed line, stable continuous waves exist. (c) We show a modified version of [8, Fig. 2.a] for comparison, where the color bar indicates the number of peaks in the solution. 

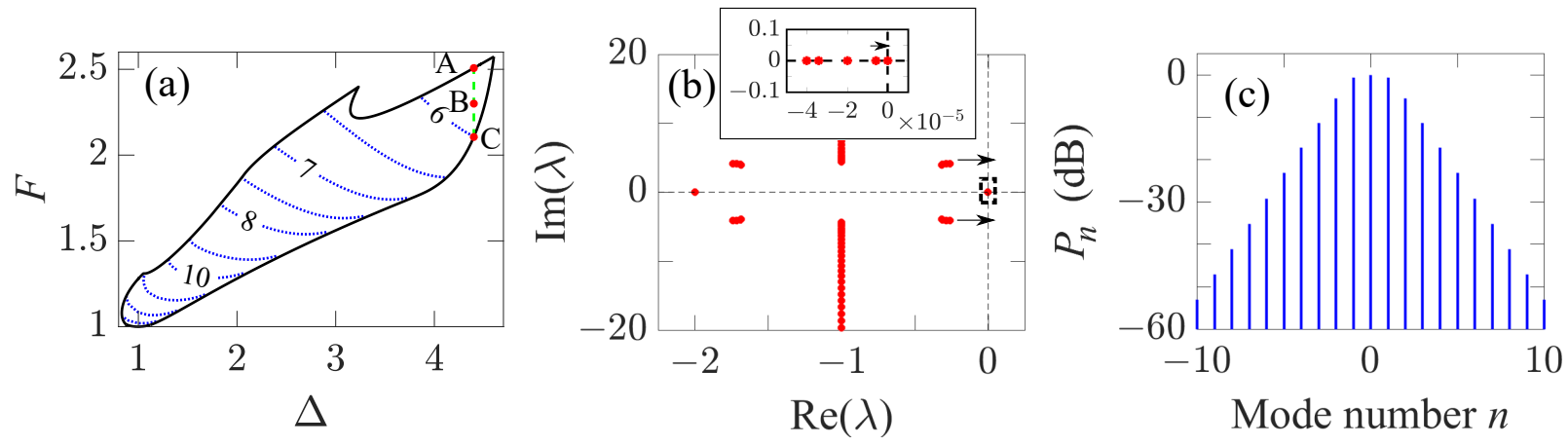

Fig. 2. (a) Stability region of the 8-period cnoidal wave at $L=50$ with a contour plot of $\gamma(\mathrm{dB})=$ $P_{n} / P_{n+1}$ at large $n$, when $P_{n}$ is the power in the $n$-th comb line. Point B corresponds to the dynamical spectrum in (b) and the Fourier spectrum in (c).

problem. In parallel, we linearize Eq. (1) and solve the linearized eigenvalue equation $\lambda \Delta \psi=\mathscr{L}\left[\psi_{s}\right] \Delta \psi$ for all its eigenvalues. The set of all the eigenvalues is the dynamical spectrum. If any eigenvalue moves to the imaginary axis, then the solution becomes unstable or ceases to exist. We then track this stability boundary, determining its location in the parameter space. Within the stability boundary, we can rapidly determine properties of the stationary solution like the comb bandwidth. This procedure is very rapid computationally and, to our knowledge, we are the only research group in optical science that has this sort of software available.

In Figs. 1(a) and (b), we show slices of the stable regions for $L=25$ and $L=50$, which we have labeled with the number of periods $N_{\text {per }}$. The case $N_{\text {per }}=1$ corresponds to solitons. For clarity, we have shown only some of the stable regions, but we solved for all the stable cnoidal waves in the parameter regions that we show. We see that the stable regions for $N_{\text {per }} / L$ approaches a constant value as $L \rightarrow \infty$, and the parameter space effectively becomes twodimensional. Below the stable cnoidal wave regions, continuous waves exist; above this region, solutions are breathers or chaotic. The stable region for solitons $\left(N_{\text {per }}=1\right)$ and some higher-period cnoidal waves almost completely overlap, which helps to explain in part why solitons are hard to access.

An important transition occurs when $\Delta \simeq 1.37$. Below this value, continuous waves become unstable when cnoidal waves become stable, and cnoidal waves can be simply accessed by raising the pump power. Above this value of $\Delta$, continuous waves remain stable when cnoidal waves are stable, and cnoidal waves can no longer be accessed by simply raising the pump power, which also helps to explain in part why solitons are hard to access. However, one can access low-period cnoidal waves deterministically, including solitons, by moving along the path shown as the dashed blue curve in Fig. 1(b) with occasional back-tracking.

In Fig. 1(c), we show the number of peaks in the final solution after jumping from an initial cnoidal wave at $\Delta=0$ and $F=6.3$ to another point in the parameter space and waiting $1.8 \mu \mathrm{s}$ [8]. In contrast to the results of the evolution equations, the boundary tracking algorithms allow us to unambiguously determine where stable solutions exist. The cnoidal wave that appears at any point along the black curves in Fig. 1(c) depends on both the initial conditions and the integration time.

In Fig. 2(a), we show the stability region for $N_{\text {per }}=8$. In Fig. 2(b), we show the dynamical spectrum for the point labeled B, and in Fig. 2(c) we show the comb spectrum with $L=50$. At point A, two eigenvalues simultaneously cross the imaginary axis, as shown by the double arrows in Fig. 2(b). At point $\mathrm{C}$, one eigenvalue hits the imaginary axis, as shown in the inset in Fig. 2(b), and the solution ceases to exist. In Fig. 2(a), we show a contour plot of the ratio $\gamma=P_{n} / P_{n+1}$-the power in the $n$-th comb line divided by the power in the $(n+1)$-th comb line when $n$ is large. For a free spectral range of $230 \mathrm{GHz}$, which corresponds to the experiments modeled in [8], we find that the 30-dB bandwidth is $22 \mathrm{THz}$, which is a large fraction of the operating frequency of $200 \mathrm{THz}$. This result suggests that it may be possible to obtain combs that are broadband, robust, and easily accessible by taking advantage of cnoidal waves.

\section{References}

1. S. A. Diddams, J. Opt. Soc. Am. B 27, B51 (2010).

2. T. J. Kippenberg, R. Holzwarth, and S. A. Diddams, Science 332, 555 (2011).

3. P. Del'Haye, A. Coillet, T. Fortier, K. Beha, D. C. Cole, K. Y. Yang, H. Lee, K. J. Vahala, S. B. Papp, and S. A. Diddams, Nat. Photonics 10, 516 (2016).

4. Z. Qi, G. D’Aguanno, and C. R. Menyuk, J. Opt. Soc. Am. B 34, 785 (2017).

5. A. Coillet, I. Balakireva, R. Henriet, K. Saleh, L. Larger, J. M. Dudley, C. R. Menyuk, and Y. K. Chembo, IEEE Photon. J. 5, 6100409 (2013).

6. A. Matsko, A. Savchenkov, W. Liang, V. Ilchenko, D. Seidel, and L. Maleki, Opt. Lett. 36, 2845 (2011).

7. S.Wang, A. Docherty, B. S. Marks, and C. R. Menyuk, J. Opt. Soc. Am. B 31, 2914 (2014).

8. J. A. Jaramillo-Villegas, X. Xue, P.-H. Wang, D. E. Leaird, and A. M. Weiner, Opt. Express 23, 9618 (2015). 